\title{
Precision Electroweak Measurements at FCC-ee
}

\author{
Elizabeth Locci ${ }^{1}$ \\ IRFU, CEA, Université Paris-Saclay \\ F-91191 Gif-sur-Yvette, France \\ E-mail: elizabeth.locciacern.ch
}

\begin{abstract}
With the discovery of the Higgs boson at the LHC in 2012, all fundamental parameters of the Standard Model Theory (SMT) have been experimentally measured. Then the global fits of the electroweak sector provide a powerful test of the internal consistency of the SMT. Any observable sensitive to electroweak corrections can be unambiguously predicted and any deviation of the measurements with respect to the predictions would reveal the existence of new, weakly interacting particles. Thus precision electroweak measurements are a key tool for constraining theories describing physics beyond the SMT. Although hadron colliders (Tevatron, LHC) have matched or occasionally exceeded the precision of LEP measurements, $\mathrm{e}^{+} \mathrm{e}^{-}$colliders are unrivalled for electroweak precision measurements. The expected performance of the future $\mathrm{e}^{+} \mathrm{e}^{-}$collider, FCC-ee, is discussed in the perspective of indirect discovery of new physics. This machine is nevertheless well-suited for direct discoveries as well.
\end{abstract}

EPS-HEP2019, European Physical Society conference on High Energy Physics

10-17 July 2019

Gent, Belgium

\section{${ }^{1}$ Speaker on behalf of the FCC Design Study Group.}




\section{Introduction}

The precision on the mass of some fundamental bricks of the SM has considerably increased since the discovery of $\mathrm{W}$ and $\mathrm{Z}$ bosons in 1983 by the UA1 and UA2 experiments. The first dramatic improvement came from the measurement of the $Z$ mass at LEP with a precision of 20 $\mathrm{MeV}$, roughly 2 orders of magnitude more accurate than the best UA1 and UA2 measurements. This measurement was then used by UA2 to derive the $\mathrm{W}$ mass from the ratio of the $\mathrm{W}$ mass to the $\mathrm{Z}$ mass, into which energy scale uncertainties cancel. The $\mathrm{W}$ mass uncertainty was then reduced by one order of magnitude. Since then this precision has constantly increased and an additional order of magnitude has been gained with the combined measurements at the Tevatron and at LHC [1].

For about 30 years, electroweak precision data have been used together with accurate Standard Model calculations to predict parameters of the theory. Electroweak radiative corrections to physical observables depend quadratically on the top-quark mass and logarithmically on the Higgs-boson mass. The global electroweak fit combines all the available electroweak measurements from various experiments into one single $\chi^{2}$ fit to provide the best evaluation of all the parameters of the Standard Model. Typical inputs are: mass and width of the $\mathrm{W}$ and $\mathrm{Z}$ boson, top-quark mass, Higgs-boson mass, Z-pole hadronic cross-section, branching ratios, asymmetries, $\tau$ polarisation, couplings. The global fits have demonstrated their impressive power in predicting the top-quark mass, which was then used into the global fit to predict the Higgs-boson mass.

With the discovery of the Higgs boson in 2012 at the LHC, the SM is now complete and, with the measurement of its mass, the electroweak sector is overconstrained. Then the global fit provides one accurate test of the internal consistency of the SM. An important consistency test of the SM is the simultaneous indirect determination of the top-quark mass and the W-boson mass.

This example illustrates the need for more precise measurements of all electroweak observables to either confirm this agreement with an unprecedented accuracy or to highlight an inconsistency that might indicate the emergence of new physics beyond the SM, as suggested by a number of theoretical and experimental arguments. After a brief description of the FCC-ee operation model, the prospects for high precision electroweak measurements at the $Z$ pole, at the W-pair production threshold and above are discussed in the next sections.

\section{The FCC-ee}

The FCC-ee [2] is the first stage of the integrated Future Circular Colliders (FCC) programme [3] at CERN. Figure 1 illustrates the baseline FCC-ee operation model with 2 interaction points (IPs) and how it compares with other options; the 4 IPs scenario is also displayed together with the ERL (Electron Recirculating Linac) option. The integrated luminosity goals for $\mathrm{W} \& \mathrm{Z}$ physics are $150 \mathrm{ab}^{-1}$ around the $\mathrm{Z}$ pole and $10 \mathrm{ab}^{-1}$ around the $\mathrm{W}$-pair production threshold. Large samples of di-boson events will also be available above the WW threshold. Current electroweak measurements will see their statistical accuracy improved by one to two orders of magnitude 
and the systematic uncertainties will have to improve to the same extent. The most critical uncertainties are those coming from the beam energy and energy spread calibration.

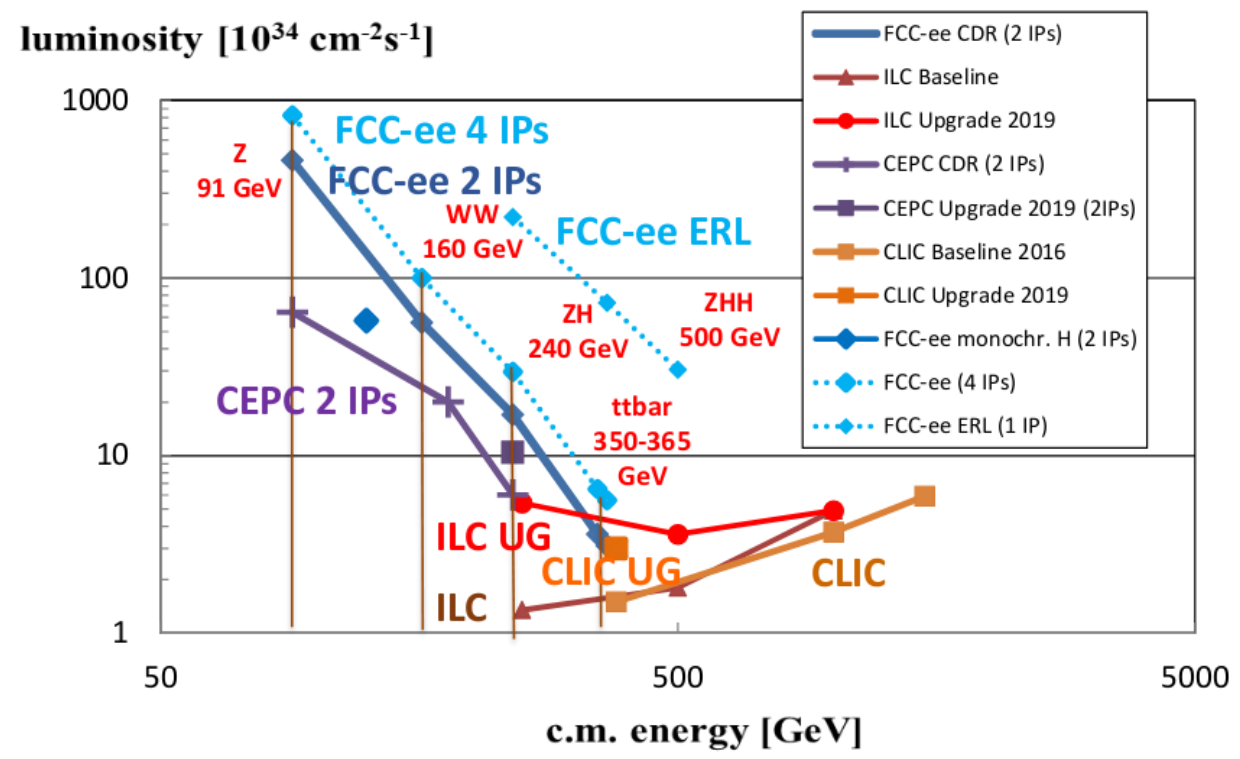

Figure 1: The FCC-ee baseline design luminosity summed over 2 IPs as a function of the centre-of mass energy $\sqrt{s}_{\mathrm{s}}$, compared to the baseline luminosities of other $\mathrm{e}^{+} \mathrm{e}^{-}$colliders. Also displayed is the FCC-ee scenario for 4 IPs and the ERL option [4] .

Beams are transversely polarized below $165 \mathrm{GeV}$ center-of-mass energy and their energies are continuously measured with resonant depolarisation on single non-colliding bunches [5]. Around the $\mathrm{Z}$ pole, $\sqrt{\mathrm{s}}_{\mathrm{s}}$ can be measured with an accuracy better than $100 \mathrm{keV}$. At the WW threshold an accuracy of $300 \mathrm{keV}$ is achievable. At higher energy, WW, ZZ and Z $\gamma$ events can be used to constrain $\sqrt{s}_{\mathrm{s}}$ with a precision of $2 \mathrm{MeV}$ and $5 \mathrm{MeV}$ at the $\mathrm{ZH}$ cross-section maximum and at the $\mathrm{t} \overline{\mathrm{t}}$ threshold respectively.

The beam crossing angle and the energy spread can be measured and monitored in the experiment itself with dimuons, which are copiously produced at all energies. Making use of energymomentum conservation, in $5 \mathrm{mn}$ the crossing angle and the energy spread can be measured with an accuracy of $0.3 \mu \mathrm{rad}$ and $0.1 \%$ respectively, provided that the angular uncertainty is kept below $100 \mu \mathrm{rad}[5]$.

The luminosity is almost irrelevant for the measurement of $\mathrm{Z}$ mass \& width, but is important for cross-section dependent measurements. The reference process is small-angle Bhabha scattering. A realistic goal for the theoretical uncertainty from higher order for low angle Bhabha is $10^{-4}$. The targeted absolute and point-to-point precisions are $10^{-4}$ and $5 \times 10^{-5}$ respectively. This requires an accuracy of $1 \mu \mathrm{m}$ on the luminometer internal radius. Clever acceptance algorithms, independent from the beam spot position, developed at LEP, should be extended to beams with crossing angle. 


\section{Physics at the $Z$ pole}

The five orders of magnitude increase of statistics with respect to LEP will yield to considerably more precise measurements of all electroweak observables. Only a selection of them is discussed here.

At LEP the $\mathrm{Z}$ mass and width were measured from the dependence of the cross-section on the center-of-mass energy. The exact choice of the off-peak energies is not as crucial at FCC-ee as it was at LEP because of the huge statistics, but has nevertheless to be optimized. As will be discussed in this section the measurement of $\alpha_{\mathrm{QED}}\left(\mathrm{m}_{\mathrm{Z}}\right)$ will drive this choice. The precise control of the beam energy and energy spread calibration, the most ctitical uncertainties, allows the $\mathrm{Z}$ mass and witdth to be measured with a precision of $100 \mathrm{keV}$ and $25 \mathrm{keV}$ respectively. Although the requirements on the detector are not crucial, the control of the acceptance, of the angular resolution at the level of $100 \mu \mathrm{rad}$, of the momentum resolution $\left(\Delta \mathrm{p}_{\mathrm{T}} / \mathrm{p}_{\mathrm{T}}{ }^{2}<4 \times 10^{-5} \mathrm{GeV}^{-}\right.$ $\left.{ }^{1}\right)$ are important.

The ratio between the hadronic and the leptonic width, $\mathrm{R}_{1}$, is a necessary input to the measurement of the lepton couplings and of $\alpha_{s}\left(\mathrm{~m}_{\mathrm{Z}}^{2}\right)$. Although systematic uncertainties largely cancel in the ratio, exploiting the FCC-ee potential requires an accurate control of the acceptance, particularly for leptons. The acceptance uncertainties, which were subdominant at LEP, need a factor 5 reduction to match the goal for a measurement at the level of $5.10^{-5}$, corresponding to an absolute uncertainty of $1.5 \times 10^{-4}$ on $\alpha_{\mathrm{s}}$. With respect to LEP the definition of the fiducial acceptance is a bit complicated by the crossing angle causing a boost in the transverse direction, which can be measured event by event for di-electrons and di-muons (section 2).

$\mathrm{Z}$ decays to individual quark flavours can be selected when the decay products can be efficiently tagged. For $Z$ decaying to $b \bar{b}$, $b$-tagging efficiency can be directly measured from data by counting single and double tag events. This is also possible, with lower performance, for c quarks. As LHC detectors and current taggers already reach 3 times the LEP b-tagging efficiency at same $\mathrm{c}$ and uds suppression in a harsher environment, a sizeable improvement is expected at FCC-ee. The systematic uncertainty from hemisphere correlations becomes dominating, however conservative FCC-ee projections expect a reduction to the level of $3 \times 10^{-4}$. Other sources such as gluon splitting and nasty sources of correlations can be studied with data at LHC.

Table 1 summarizes the estimated accuracy for the normalized partial widths and the expected improvement with respect to LEP.

\begin{tabular}{lccc}
\hline \hline & Statistical uncertainty & Systematic uncertainty & improvement w.r.t. LEP \\
\hline$R_{\mu}\left(R_{\ell}\right)$ & $10^{-6}$ & $5 \times 10^{-5}$ & 25 \\
$R_{\tau}$ & $1.5 \times 10^{-6}$ & $10^{-4}$ & 20 \\
$R_{\mathrm{e}}$ & $1.5 \times 10^{-6}$ & $3 \times 10^{-4}$ & 20 \\
$R_{\mathrm{b}}$ & $1.5 \times 10^{-6}$ & $3 \times 10^{-4}$ & 10 \\
$R_{\mathrm{c}}$ & $1.5 \times 10^{-4}$ & $15 \times 10^{-4}$ & 10 \\
\hline
\end{tabular}

Table 1: Relative precision on the normalized $\mathrm{Z}$ partial widths $\left(\mathrm{R}_{\mathrm{f}}\right)$ at FCC-ee (relative uncer- 
tainties). Expected statistical and systematic precisions for $150 \mathrm{ab}^{-1}$ are shown. The last column highligts the improvement on precision with respect to LEP [6].

For unpolarized electron beams the forward-backward asymmetry, $\mathrm{A}_{\mathrm{FB}}$, depends on both initial and final states couplings. For leptonic final states $A_{F B}$ quadratically depends on $\sin ^{2} \theta_{\text {eff, }}$ which can be extracted with a precision of $5 \times 10^{-6}$ at least. Tau polarization can be measured from the angular distributions and momenta of the decay products and provides a simultaneous determination of $A_{e}$ and $A_{\tau}$ and thus of $\sin ^{2} \theta_{\text {eff, }}$, with $6 \times 10^{-6}$ accuracy. Also $b$ and $c$ forwardbackward asymmetries can be used to provide inputs to quark couplings. Table 2 summarizes the estimated accuracy for the coupling ratio factors, with the expected improvement with respect to LEP. The expected precision on the vector and axial neutral couplings is shown in Table 3.

\begin{tabular}{lccc}
\hline \hline & Statistical uncertainty & Systematic uncertainty & improvement w.r.t. LEP \\
\hline $\mathcal{A}_{e}$ & $5 . \times 10^{-5}$ & $1 . \times 10^{-4}$ & 50 \\
$\mathcal{A}_{\mu}$ & $2.5 \times 10^{-5}$ & $1.5 \times 10^{-4}$ & 30 \\
$\mathcal{A}_{\tau}$ & $4 . \times 10^{-5}$ & $3 . \times 10^{-4}$ & 15 \\
$\mathcal{A}_{b}$ & $2 \times 10^{-5}$ & $30 \times 10^{-4}$ & 5 \\
$\mathcal{A}_{c}$ & $3 \times 10^{-4}$ & $80 \times 10^{-4}$ & 4 \\
\hline $\sin ^{2} \theta_{W, \text { eff }}$ (from muon FB) & $10^{-7}$ & $2-5 \times 10^{-6}$ & 100 \\
$\sin ^{2} \theta_{W, \text { eff }}$ (from tau pol) & $10^{-7}$ & $6.6 \times 10^{-6}$ & 75 \\
\hline
\end{tabular}

Table 2: Precision on the coupling-ratio factors $A_{f}$ for various fermions at FCC-ee. Expected statistical and systematic precisions for $150 \mathrm{ab}^{-1}$ are shown (relative uncertainties). The last column highligts the improvement on precision with respect to LEP. The last two rows show the expected precision (absolute uncertainties) on $\sin ^{2} \theta_{\mathrm{W}, \text { eff }}$ from the measurement of the muon forward asymmetry and tau polarization, respectively [6].

\begin{tabular}{lcc}
\hline \hline fermion type & $g_{a}$ & $g_{v}$ \\
\hline $\mathrm{e}$ & $1.5 \times 10^{-4}$ & $2.5 \times 10^{-4}$ \\
$\mu$ & $2.5 \times 10^{-5}$ & $2 . \times 10^{-4}$ \\
$\tau$ & $0.5 \times 10^{-4}$ & $3.5 \times 10^{-4}$ \\
$\mathrm{~b}$ & $1.5 \times 10^{-3}$ & $1 \times 10^{-2}$ \\
$\mathrm{c}$ & $2 \times 10^{-3}$ & $1 \times 10^{-2}$ \\
\hline
\end{tabular}

Table 3: Expected precision on fermion axial and vector neutral couplings to the $Z$ (relative uncertainties), computed from the projected uncertainties on the normalized $Z$ partial widths $\left(R_{f}\right.$ ) given in Table 1 combined with the projections on coupling-ratio factors shown in Table 2 [6].

The current estimation of $\alpha_{\mathrm{QED}}\left(\mathrm{m}_{\mathrm{Z}}^{2}\right)$ is based on the precise measurement $\left(10^{-10}\right)$ of $\alpha_{\text {QED }}$ at zero momentum transfer extrapolated to the Z-mass scale with a $1.1 \times 10^{-4}$ uncertainty [7], dominated by the experimental determination of the hadronic vacuum polarization. It was demonstrated [8] that $\mathrm{A}_{\mathrm{FB}}(\mu \mu)$ around the $\mathrm{Z}$ pole could also be used to directly measure the electromagnetic coupling constant, $\alpha_{\mathrm{QED}}\left(\mathrm{m}^{2} \mathrm{z}\right)$, with an adequate 
accuracy to match the precision on the other input parameters of the Standard Model and exploiting the full potential of the FCC to constrain or fit the parameters of Beyondthe-Standard Model (BSM) theories. The relative uncertainty on $\alpha_{\mathrm{QED}}$, proportional to the relative uncertainty on $\mathrm{A}_{\mathrm{FB}}(\mu \mu)$, is minimal at the two centre-of mass energies 87.9 and $94.3 \mathrm{GeV}$. As $\mathrm{A}_{\mathrm{FB}}(\mu \mu)$ changes sign on the opposite sides of the $\mathrm{Z}$ pole, most sources of uncertainties cancel by combining the measurements at these two centre-ofmass energies and a relative statistical accuracy of $3 \times 10^{-5}$ is achievable. The knowledge of the centre-of-mass energy is the dominant source of experimental systematic uncertainty. Missing electroweak orders are estimated to contribute to the dominant theoretical systematic uncertainty at the level of a few $10^{-4}$, and a factor 10 improvement is required to match the experimental contribution to the total uncertainty on $\alpha_{\mathrm{QED}}$.

\section{Physics at the WW production threshold}

The large number of $\mathrm{W}$ pairs collected at the WW production threshold will provide precise measurements of $\mathrm{W}$ boson properties.

A precise direct determination of the $\mathrm{W}$ mass can be achieved by observing the rapid rise of the $\mathrm{W}$-pair production cross-section near its kinematic threshold [6]. At LEP the $\mathrm{W}$ mass was measured with a single energy point with an accuracy of $210 \mathrm{MeV}$. The sensitivity of the crosssection to mass and width varies with the center-of-mass energy, then their simultaneous measurement may be optimized by carefully choosing 2 energy points. The mass and width can also be extracted simultaneously from a 2-dimensional fit. A scan of all possible couples of $\sqrt{ }_{\mathrm{s}}$ and fraction of luminosity at the highest energy point has pointed to an optimal couple and fraction leading to a $0.62 \mathrm{MeV}$ uncertainty on the mass and $1.5 \mathrm{MeV}$ on the width. The requirements on the control of the beam energy and of the luminosity are similar to those already discussed at the $\mathrm{Z}$ pole.

The $\mathrm{W}$ mass and width can also be determined from the kinematic reconstruction of the $\mathrm{W}$-pair products at the three center-of-mass energies [9]. Both fully hadronic and semi-leptonic channels are used. At all energies the statistical uncertainty is below $1 \mathrm{MeV}$. At the $\mathrm{W}$-pair production threshold the beam energy can be measured with an accuracy of $300 \mathrm{keV}$ through resonant depolarisation. At higher energies $\mathrm{Z} \gamma \& \mathrm{ZZ}$ events are needed to control the beam energy. The ultimate goal is a simultaneous fit of $\mathrm{WW}, \mathrm{ZZ}$, and $\mathrm{Z} \gamma$ events to extract the mass ratio with potential large cancellations of systematic uncertainties.

The leptonic and hadronic branching ratios were measured at LEP and a 2.8 sigma excess of $\tau$ leptons was observed. A large collection of $\mathrm{W}$ decays will provide a clarification on this discrepancy as shown in Table 4, where the expected precision for each decay mode at the FCC-ee is compared to LEP2. 


\begin{tabular}{|c|c|c|c|c|}
\hline Decay mode relative precision & $B(\mathrm{~W} \rightarrow \mathrm{e} \nu)$ & $B(\mathrm{~W} \rightarrow \mu \nu)$ & $B(\mathrm{~W} \rightarrow \tau \nu)$ & $B(\mathrm{~W} \rightarrow q q)$ \\
\hline LEP2 & $1.5 \%$ & $1.4 \%$ & $1.8 \%$ & $0.4 \%$ \\
FCC-ee & $3 \cdot 10^{-4}$ & $3 \cdot 10^{-4}$ & $4 \cdot 10^{-4}$ & $1 \cdot 10^{-4}$ \\
\hline
\end{tabular}

Table 4: Relative precision on the determination of the $\mathrm{W}$ decay branching ratios. Combined results with LEP2 data are compared to the projected precision obtainable with FCC-ee. [6].

Within the Standard Model, the hadronic branching ratio is related to the Cabibbo-KobayashiMaskawa (CKM) quark mixing matrix and to the strong coupling constant $\alpha_{\mathrm{s}}$. Then assuming CKM unitarity, measuring the hadronic branching fraction with a relative accuracy of $10^{-4}$ allows the extraction of $\alpha_{s}$ with an absolute precision of $2 \times 10^{-4}$. If $\alpha_{s}$ is obtained from another independent measurement, this same relation can be used to test the CKM unitarity at the precision level of a few $10^{-4}$.

The flavour tagging of jets from $\mathrm{W}$ decays can be exploited to perform a measurement of the $\mathrm{W}$ coupling to $\mathrm{b}$ and $\mathrm{c}$ quarks. Also rare $\mathrm{W}$ decays can be probed at the level of $10^{-7}$ probability.

To reach this level of accuracy in the above measurements, an excellent control of jet reconstruction and lepton identification is needed to master cross-contaminations between leptonic channels.

The determination of the triple gauge couplings has very important implications on BSM physics. At LEP2 the TGCs were constrained to the level of a few \%. At the FCC-ee, the diboson process can be measured at several different energies with a much higher luminosity. In the model-independent framework of the effective field theory, the Standard Model Lagrangian is supplemented by higher dimension operators. At large enough energy scale, the leading effects of new physics at the electroweak scale are well parametrized by dimension-six operators and we will focus on CP-even dimension-six operators. Then the Lagrangian can be written as a function of the TGCs, which can be reduced to three anomalous TGCs by imposing gauge invariance constraint. The differential WW cross-section can be parametrized by the production polar angle and the two decay angles for each $\mathrm{W}$. Using only semi-leptonic decays, a simultaneous fit of the three TGCs has been performed, as well as individual fits assuming the other two TGCs are zero, with three different scenarii, and the $68 \%$ confidence level is shown on Figure 6.

The three anomalous TGCs can be mapped to the three operators: $\mathrm{O}_{\mathrm{HW}}, \mathrm{O}_{\mathrm{HB}}$ and $\mathrm{O}_{3 \mathrm{~W}}$. Then the precision on the anomalous couplings can be translated to the reach for the new physics energy scale $\Lambda$. Assuming that the couplings of the operators, $\mathrm{c}_{\mathrm{i}}$, is one, the TGCs measurements are sensitive to multi $\mathrm{TeV}$ new physics scales.

\section{Conclusions}

The FCC-ee has a considerable physics potential. With the huge amount of Zs and W pairs produced, a large number of electroweak observables will be measured with an unprecedented statistical precision ( 1 or 2 orders of magnitude with respect to present measurements).

In order to fully exploit this potential, the systematic uncertainty must match the statistical uncertainty. The beam energy calibration is the dominant source of systematic uncertainty for a 
number of observables, however the center-of-mass energy can be measured with an accuracy of $100 \mathrm{keV}$ around the $\mathrm{Z}$ pole and $300 \mathrm{keV}$ around the $\mathrm{W}$-pair production threshold; other effects such as beam energy spread and asymmetry for instance are under control at the required level. The luminosity uncertainty is critical for all measurements related to the $\mathrm{Z}$ cross-section. The expected absolute accuracy is $10^{-4}$ and the relative uncertainty is $10^{-5}$; to reach this expectation, the precision of construction and metrology of the luminometer internal radius must be controlled at the level of the $\mu \mathrm{m}$. Also required are the control of acceptance, lepton identification, good photon $/ \pi^{0}$ separation, flavour-tagging.

\section{Acknowledgements}

I wish to thank all the contributors to the preparation of the talk, particularly Roberto Tenchini.

\section{References}

[1] M. Tanabashi et al. (Particle Data Group) , Phys.Rev. D98, 030001 (2018) and 2019 update.

[2] M. Benedikt, A. Blondel, O. Brunner, M. Capeans Garrido, F. Cerutti, J. Gutleber et al., Future Circular Collider, Vol. 2, The Lepton Collider (FCC-ee), Eur.Phys.J. ST228 (2019) no.2, 261-623, doi:10.1140/epjcst/e2019-900045-4.

[3] M. Benedikt, A. Blondel, O. Brunner, M. Capeans Garrido, F. Cerutti, J. Gutleber et al.,Future Circular Collider - European Strategy Update Documents: The FCC integratedprogramme (FCCint), https://cds.cern.ch/record/2653673, Jan, 2019.

[4] F. Zimmermann, Talk given at the FCC Week 2019, 24-28 June 2019.

[5] A. Blondel et al., Polarization and centre-of-mass energy calibration at FCC-ee, arXiv:1909.12245 [physics.acc-ph], Sep 26, 2019.

[6] A. Blondel, C. Grojean, P. Janot, M. McCullough et al. , FCC-ee Physics, Experiments and Detectors, in preparation.

[7] J. Erler, A. Freitas, Electroweak Model and Constraints on New Physics, in K.A. Olive et al. (PDG), Chin. Phys. C38 (2014) 090001, doi : 10 .1088/1674-1137/38/9/090001.

[8] P. Janot, Direct measurement of $\alpha_{Q E D}\left(\mathrm{~m}^{2} \mathrm{z}\right)$ at the FCC, JHEP 1602 (2016) 053, arXiv:1512.05544[hep-ph], doi:10.1007/JHEP02(2016)053.

[9] M. Béguin, talk presented at EPS-HEP2019, these proceedings. 